\title{
Quality of Life before and after Endoscopic Pituitary Surgery as Measured by the Short-Form-36
}

\author{
Edward C. Kuan ${ }^{1}$ Frederick Yoo ${ }^{1} \quad$ Jennifer Chyu ${ }^{1} \quad$ Angela Oh $^{1} \quad$ Marvin Bergsneider $^{2}$ Marilene B. Wang ${ }^{1}$ \\ ${ }^{1}$ Department of Head and Neck Surgery, David Geffen School of \\ Medicine at the University of California, Los Angeles (UCLA) Medical \\ Center, Los Angeles, California, United States \\ ${ }^{2}$ Department of Neurosurgery, David Geffen School of Medicine at

\begin{abstract}
Address for correspondence Marilene B. Wang, MD, Department of Head and Neck Surgery, David Geffen School of Medicine at UCLA, 10833 Le Conte Avenue, CHS 62-132, Los Angeles, CA 90095-1624, United States (e-mail: mbwang@ucla.edu).
\end{abstract} the University of California, Los Angeles (UCLA) Medical Center, Los Angeles, California, United States

J Neurol Surg B 2018;79:314-318.

\begin{abstract}
Keywords

- pituitary surgery

- quality of life

- outcomes

- skull base surgery

- SF-36

Objectives/Hypotheses To assess quality of life (QOL) after transnasal, endoscopic pituitary surgery using the 36-item short form (SF-36) instrument.

Design Retrospective review was used for this study.

Setting The study was conducted in a tertiary academic medical center.

Participants Patients who underwent endoscopic, transnasal, transsphenoidal surgery for pituitary adenomas between January 1, 2007 and July 1, 2016 and completed preoperative and postoperative SF-36 surveys.

Main Outcome Measures SF-36 survey data as measured by its eight domains (physical functioning, physical role functioning, emotional role functioning, energy/ fatigue, emotional well-being, social functioning, pain, and general health).

Results There were 18 preoperative, 13 short-term ( 2 weeks or less after surgery) postoperative, and 14 longer term ( $>2$ weeks after surgery) postoperative surveys. There was no significant difference between preoperative and long-term postoperative SF-36 scores across domains $(p>0.05)$. In comparing short-term postoperative and preoperative scores, tumor size was positively associated with emotional well-being ( $p=0.049)$ and general health scores $(p=0.031)$, while visual changes preoperatively were positively associated with general health scores $(p=0.046)$. Compared with standard U.S. general population summary data, these patients scored lower preoperatively in all domains except for emotional role functioning and pain $(p<0.05)$. Postoperatively, patients improved to baseline general population data scores with the exception of the physical role functioning domain $(p<0.0001)$.

Conclusion Patients undergoing endoscopic, transnasal, transsphenoidal surgery for pituitary adenomas had lower QOL in six of eight domains preoperatively, but improved to baseline values on the long run after surgery in seven of eight domains. This suggests that minimally invasive pituitary surgery has a restorative role in general $\mathrm{QOL}$ as measured by the SF-36.
\end{abstract}

received

April 12, 2017

accepted after revision

September 19, 2017

published online

November 7, 2017 (c) 2018 Georg Thieme Verlag KG

Stuttgart · New York
DOI https://doi.org/ 10.1055/s-0037-1608648. ISSN 2193-6331. 


\section{Introduction}

As endoscopic techniques have gained prominence for selective anterior skull base surgery indications, many skull base pathologies, particularly pituitary tumors, may be safely and effectively managed via transnasal, endoscopic approaches. Of utmost importance in endoscopic skull base surgery is the frequent need to surgically manipulate or resect normal, uninvolved, and noninflamed intranasal structures to gain adequate access for a functional surgical corridor. In doing so, iatrogenic sinonasal sequelae, such as crusting, rhinosinusitis, epistaxis, or hyposmia, become part of the disease burden of such patients. Thus, in following a rising trend in outcomes following endoscopic surgery in rhinology, an active area of investigation is related to quality of life (QOL) outcomes following skull base surgery. ${ }^{1-3}$

The vast majority of QOL studies are performed using the 22item Sinonasal Outcomes Test (SNOT-22), which focuses on sinonasal symptoms but also has applications in sleep and psychological symptoms. ${ }^{4}$ Within skull base surgery, various QOL outcomes instruments are available, generally focusing on performance status, pain, site-specific symptoms, and psychological metrics. ${ }^{5-8}$ A commonly used instrument with experience in otolaryngological and neurosurgical conditions is the validated 36-item Short Form health survey questionnaire (SF-36). ${ }^{9}$ Abridged versions of this instrument have been utilized in part for assessment of QOL outcomes following skull base surgery, but comprehensive assessment of general health status using the SF-36 has not been performed before. In this study, we utilize the SF-36 to evaluate general health QOL outcomes following endoscopic pituitary surgery for adenomas.

\section{Methods}

Institutional Review Board approval was obtained for this study. We performed a retrospective chart review of all patients who underwent endoscopic, transnasal surgery at a tertiary academic medical center for pituitary adenomas between January 1, 2007 and July 1, 2016. All patients who completed preoperative and postoperative SF-36 questionnaires were included in the analysis. Demographic variables including age, gender, height, weight, body-mass index (BMI), tumor size and characteristics (functioning versus non-functioning), primary versus recurrent lesion, and presenting symptoms (i.e., headaches, visual changes) were also collected. Additionally, reconstructive technique, surgical complications (e.g., cerebrospinal fluid [CSF] leak), eventual recurrence, and need for adjuvant radiation therapy were also reviewed. All patients were treated via an endoscopic, transnasal, transsphenoidal approach as typically performed by a skull base surgery team comprised of one otolaryngologist (M.B.W.) and one neurosurgeon (M.B.). ${ }^{2}$ The middle turbinate is spared in all cases, with the surgical corridor primarily consisting of bilateral wide sphenoidotomies.

The SF-36 is a 36-item patient-reported health outcomes questionnaire developed by Ware et al as part of the Medical Outcomes Study. ${ }^{9}$ Patient responses can be quantified and converted into eight separate domains (physical functioning, role limitations due to physical problems, role limitations
Table 1 Baseline general population SF-36 domain scores as reported by the medical outcomes study $(n=2,474)^{9}$

\begin{tabular}{|l|c|}
\hline Domain & Mean \pm SD \\
\hline Physical functioning & $84.2 \pm 23.3$ \\
\hline Physical role functioning & $80.9 \pm 34.0$ \\
\hline Emotional role functioning & $81.3 \pm 33.0$ \\
\hline Energy/fatigue & $60.9 \pm 20.9$ \\
\hline Emotional well-being & $74.7 \pm 18.1$ \\
\hline Social functioning & $83.3 \pm 22.7$ \\
\hline Pain & $75.2 \pm 23.7$ \\
\hline General health & $71.9 \pm 20.3$ \\
\hline
\end{tabular}

Abbreviation: SD, standard deviation.

due to emotional problems, energy and fatigue, emotional well-being, social functioning, pain, general health status). The reliability, central tendency, and variability of each of these domains were reported based on a survey of 2,474 individuals, and served as the baseline U.S. population values to which patient responses can be compared (- Table 1). ${ }^{9}$

Three groups at separate times were compared: preoperative, short-term postoperative ( $\leq 2$ weeks), and longer term postoperative ( $>2$ weeks), which reflects recovery time beyond the early postoperative period. Statistical analysis was performed using independent two-tailed Student's $t$-tests for comparing two variables or one-way analysis of variances for more than two variables with a significance level of 0.05 . Additionally, absolute differences between short-term postoperative and preoperative, as well as between long-term postoperative and preoperative SF-36 scores were calculated and used as an outcome measure. Univariate linear regression was thus performed using the above applicable risk factors whenever applicable to determine predictors of SF-36 score changes.

\section{Results}

There were 18 patients who completed 18 preoperative, 13 short-term postoperative, and 14 longer term postoperative surveys. The range for the longer term follow-up cohort was 20 to 244 days (mean $87 \pm 76$ ). The mean age was $45.6 \pm 16.2$ years (range, 16-72). Thirty nine percent ( 7 of 18 ) were male patients. The average height and weight were $65.3 \pm 4.8$ inches and $81.4 \pm 18.9 \mathrm{~kg}$, respectively. Mean BMI was $29.0 \pm 5.9$. The mean tumor size was $15.1 \pm 11.3 \mathrm{~mm}$. Regarding tumor functional characteristics, five (27\%) were acromegalic, five (27\%) suffered from Cushing's disease, one (6\%) suffered from a prolactinoma, and the remaining seven (39\%) were nonfunctioning. Five (27\%) patients presented with visual changes, while two (12\%) presented with headaches. One (6\%) patient had a previous recurrent lesion and was thus undergoing revision surgery. Three (17\%) patients required reconstruction using a nasoseptal flap due to intraoperative CSF leak. No patients required adjuvant radiation. No surgical complications, including no postoperative CSF leaks or recurrences, occurred in any patient for at least a year after surgery follow-up time. 
There was no significant difference between preoperative and long-term postoperative SF-36 scores across all domains (all $p>0.05$, - Table 2). No significant differences were noted between preoperative and short-term postoperative scores; there was a trend toward decreased physical role functioning for the short-term postoperative group as compared with preoperative scores, but this was not significant $(p=0.06)$. Compared with baseline U.S. population summary data, patients with pituitary adenomas scored lower preoperatively in all domains except for emotional role functioning and pain (-Table 2). Postoperatively, at greater than 2 weeks postoperatively, these patients improved to baseline general population data scores with the exception of the physical role functioning domain $(p<0.0001)$. In comparing those patients who underwent sellar reconstruction using nasoseptal flaps versus those who did not, there were no differences among preoperative, short-term, and longer term QOL outcomes in any domain $(p<0.05)$.

In the regression analysis, no significant predictors of longer term postoperative score changes were identified. However, in comparing short-term postoperative and preoperative scores, tumor size was positively associated with both emotional well-being $(p=0.049)$ and general health $(p=0.031)$ scores, while visual changes preoperatively were positively associated with general health scores $(p=0.046)$. Headache at presentation was negatively associated with emotional role functioning scores $(p=0.006)$.

\section{Discussion}

With a large proportion of pituitary adenomas now being approached endoscopically, skull base surgeons now have the unique opportunity to study both short- and longer term QOL outcomes across multiple domains. There has been some investigation on the effects of a tumor diagnosis on
QOL. For instance, Gil et al reported that $74 \%$ of patients had improved QOL following open anterior skull base surgery for malignancy, ${ }^{10}$ with emotional domains seeming to reliably improve following the early postoperative period. ${ }^{11}$ These results are consistent with our findings with the SF-36, where emotional well-being and emotional role functioning scores improved postoperatively. Needless to say, a tumor diagnosis is a stressful situation and likely has a negative effect on QOL for afflicted patients. Timely treatment and "putting the diagnosis behind them" is thus an effective strategy for these patients.

Specific to pituitary surgery, QOL following endoscopic surgery is generally preserved or even improved. ${ }^{12}$ However, extended endonasal approaches, which are sometimes necessary for wider access due to primary tumor burden and extent of involvement, tend to produce worse QOL outcomes when compared with standard targeted endoscopic pituitary approaches, likely due to dissection of nonpathologic anatomy or being associated with a higher risk of CSF leak. ${ }^{13}$ Also, primary tumor factors, such as large tumor size and compressive injury of surrounding structures (e.g., optic chiasm), can lead to further decline in QOL due to symptom manifestations (e.g., visual loss, headache, endocrinopathy). In addition, adjuvant therapy for more extensive sellar lesions, such as radiation therapy, though infrequent, may further contribute to sinonasal morbidity. Limiting dissection to the immediate surrounding surgical area whenever possible has been shown to be associated with improved sinonasal symptom scores ${ }^{2}$ and preservation of native anatomy may decrease the risk of patients developing postoperative radiographic sinusitis. ${ }^{14}$ Though no certain link has been established through studies, there is some evidence suggesting that sinonasal symptoms and overall health status are correlated, as outcome scores are generally worse in the shortterm postoperatively ( $<2$ weeks), but recover to baseline by

Table 2 Preoperatively and short- and long-term postoperative SF-36 scores for patients with pituitary adenomas undergoing endoscopic pituitary surgery

\begin{tabular}{|l|l|l|l|l|l|l|l|}
\hline \multirow{2}{*}{ Domain } & \multicolumn{6}{|l|}{ Preoperative $(\boldsymbol{n}=\mathbf{1 8})$} & \multicolumn{3}{l|}{ ST postoperative $(\boldsymbol{n}=13)$} & \multicolumn{2}{l|}{ LT postoperative $(\boldsymbol{n}=14)$} \\
\cline { 2 - 8 } & Mean \pm SD & $\boldsymbol{p}$ (vs. MOS) & Mean \pm SD & $\boldsymbol{p}$ (vs. Pre-op) & Mean \pm SD & $\boldsymbol{p}$ (vs. Pre-op) & $\boldsymbol{p}$ (vs. MOS) \\
\hline $\begin{array}{l}\text { Physical } \\
\text { functioning }\end{array}$ & $68.3 \pm 29.9$ & $0.004^{\mathrm{a}}$ & $61.9 \pm 35.1$ & 0.59 & $72.1 \pm 33.4$ & 0.74 & 0.05 \\
\hline $\begin{array}{l}\text { Physical role } \\
\text { functioning }\end{array}$ & $58.3 \pm 45.4$ & $0.005^{\mathrm{a}}$ & $26.9 \pm 43.9$ & 0.06 & $41.1 \pm 43.4$ & 0.29 & $<0.0001^{\mathrm{a}}$ \\
\hline $\begin{array}{l}\text { Emotional role } \\
\text { functioning }\end{array}$ & $66.7 \pm 42.8$ & 0.06 & $64.1 \pm 48.0$ & 0.88 & $81.0 \pm 36.3$ & 0.33 & 0.97 \\
\hline Energy/fatigue & $39.4 \pm 25.9$ & $<0.0001^{\mathrm{a}}$ & $40.0 \pm 24.8$ & 0.95 & $53.9 \pm 29.6$ & 0.15 & 0.21 \\
\hline $\begin{array}{l}\text { Emotional } \\
\text { well-being }\end{array}$ & $66.2 \pm 18.6$ & $0.05^{\mathrm{a}}$ & $70.0 \pm 19.9$ & 0.59 & $75.4 \pm 18.3$ & 0.17 & 0.89 \\
\hline $\begin{array}{l}\text { Social } \\
\text { functioning }\end{array}$ & $70.8 \pm 29.7$ & $0.02^{\mathrm{a}}$ & $65.4 \pm 15.4$ & 0.55 & $76.8 \pm 28.1$ & 0.57 & 0.29 \\
\hline Pain & $68.9 \pm 21.1$ & 0.26 & $59.6 \pm 22.5$ & 0.25 & $72.7 \pm 25.7$ & 0.65 & 0.69 \\
\hline General health & $61.9 \pm 21.2$ & $0.04^{\mathrm{a}}$ & $67.7 \pm 24.7$ & 0.49 & $70.0 \pm 20.8$ & 0.29 & 0.73 \\
\hline
\end{tabular}

Abbreviations: LT, long term; MOS, medical outcomes study; SD, standard deviation; ST, short term.

Note: Baseline general population values are reported for comparison.

${ }^{\text {a }}$ Statistical significance at the 0.05 level. 
3 months. ${ }^{15,16}$ With this study cohort, all domains improved to baseline population levels after 2 weeks postoperatively with the exception of the physical role functioning domain. Although no longer term follow-up (e.g., > 3 months) is available, we anticipate that in the uncomplicated pituitary adenoma case, all domains should improve after a longer interval as part of expected postoperative recovery.

A few studies have assessed QOL outcomes in endoscopic pituitary surgery using the SF-36. One series from Canada utilizing the SF-36 examined 54 patients who had previously undergone endoscopic pituitary surgery. ${ }^{17}$ Compared with the general Canadian population, all domain scores returned to baseline except for a significantly lower general health domain score. ${ }^{17}$ Another study from Japan compared SF-36 scores of patients with pituitary macroadenomas, who underwent endoscopic surgery preoperatively and at 1 and 6 months postoperatively, and found that physical scores decreased in the short term but improved thereafter, while mental scores persistently remained improved postoperatively. ${ }^{18}$ Finally, a prospective study of 55 patients found that there was no significant difference in QOL between preoperative and 1 year postoperative domain scores. ${ }^{19}$ Taken together, these findings paralleled those in this study and once again underscored that preservation or improvement of QOL is possible with surgery.

We found positive associations between short-term postoperative SF-36 scores and both preoperative tumor size and visual changes. The interpretation of this is that larger tumors tend to exhibit greater mass effect and may thus be more symptomatic, which negatively affects quality of life. Tumor-related visual changes are similarly difficult for patients to manage. Thus, in the short run, surgical resection of symptomatic tumors should naturally result in improved QOL. Moreover, there is evidence from prior studies that suggests worse or no impact on QOL outcomes with flap reconstruction. ${ }^{20-22}$ In this study, we found no differences in preoperative, short-term postoperative, and longer term postoperative SF-36 scores between those patients who had nasoseptal flap reconstruction and those who did not.

There are a few limitations to this study. First, pituitary adenomas, though considered as neoplastic disease, are benign tumors that, in general, can be removed reliably via a minimally invasive approach in most cases. Therefore, the expectation is that there is limited long-term impact on QOL. Second, the sample size for the study is rather low, but does appear to provide sufficient data for meaningful comparison to the general population. For the same reason, it is difficult to provide subgroup analyses of meaning (e.g., differences in QOL amongst functional and nonfunctional tumors). Third, use of the SF-36 as an outcome instrument provides a partially-quantifiable measure of general health status, which may not necessarily correspond to surgery-specific health factors (e.g., QOL changes due to being under general anesthesia). Fourth, though this study cohort encompasses follow-up data beyond the immediate postoperative period of 1 to 2 weeks, there are ultimately limited data on true long-term follow-up, which is on the order of months to a year after surgery.

\section{Conclusion}

Patients undergoing endoscopic, transnasal, transsphenoidal surgery for pituitary adenomas initially have lower QOL in 6 of 8 domains preoperatively, but improved to baseline values after the early postoperative period in 7 of 8 domains when compared with the general population. This suggests that minimally invasive pituitary surgery has a restorative role in general QOL as measured by the SF-36.

Note

This study was presented as a poster at the 26th Annual Meeting of the North American Skull Base Society, March 3-5, 2017, in New Orleans, Los Angeles, United States.

Funding

None.

Conflict of Interest

None.

\section{References}

1 Greig SR, Cooper TJ, Sommer DD, Nair S, Wright ED. Objective sinonasal functional outcomes in endoscopic anterior skull-base surgery: an evidence-based review with recommendations. Int Forum Allergy Rhinol 2016;6(10):1040-1046

2 Thompson CF, Suh JD, Liu Y, Bergsneider M, Wang MB. Modifications to the endoscopic approach for anterior skull base lesions improve postoperative sinonasal symptoms. J Neurol Surg B Skull Base 2014;75(01):65-72

3 Little AS, Jahnke H, Nakaji P, Milligan J, Chapple K, White WL. The anterior skull base nasal inventory (ASK nasal inventory): a clinical tool for evaluating rhinological outcomes after endonasal surgery for pituitary and cranial base lesions. Pituitary 2012;15(04): 513-517

4 Hopkins C, Gillett S, Slack R, Lund VJ, Browne JP. Psychometric validity of the 22-item Sinonasal Outcome Test. Clin Otolaryngol 2009;34(05):447-454

5 Gil Z, Abergel A, Spektor S, Shabtai E, Khafif A, Fliss DM. Development of a cancer-specific anterior skull base quality-of-life questionnaire. J Neurosurg 2004;100(05):813-819

6 de Almeida JR, Vescan AD, Gullane PJ, et al. Development of a disease-specific quality-of-life questionnaire for anterior and central skull base pathology-the skull base inventory. Laryngoscope 2012;122(09):1933-1942

7 de Almeida JR, Witterick IJ, Gullane PJ, et al. Quality of life instruments for skull base pathology: systematic review and methodologic appraisal. Head Neck 2013;35(09):1221-1231

8 Little AS, Kelly D, Milligan J, et al. Prospective validation of a patientreported nasal quality-of-life tool for endonasal skull base surgery: The Anterior Skull Base Nasal Inventory-12. J Neurosurg 2013; 119(04):1068-1074

9 Ware JE Jr, Sherbourne CD. The MOS 36-item short-form health survey (SF-36). I. Conceptual framework and item selection. Med Care 1992;30(06):473-483

10 Gil Z, Abergel A, Spektor S, et al. Quality of life following surgery for anterior skull base tumors. Arch Otolaryngol Head Neck Surg 2003;129(12):1303-1309

11 Abergel A, Fliss DM, Margalit N, Gil Z. A prospective evaluation of short-term health-related quality of life in patients undergoing anterior skull base surgery. Skull Base 2010;20(01): 27-33 
12 McCoul ED, Bedrosian JC, Akselrod O, Anand VK, Schwartz TH. Preservation of multidimensional quality of life after endoscopic pituitary adenoma resection. J Neurosurg 2015;123(03):813-820

13 Alobid I, Enseñat J, Mariño-Sánchez F, et al. Expanded endonasal approach using vascularized septal flap reconstruction for skull base tumors has a negative impact on sinonasal symptoms and quality of life. Am J Rhinol Allergy 2013;27(05):426-431

14 Deconde AS, Vira D, Thompson CF, Wang MB, Bergsneider M, Suh JD. Radiologic assessment of the paranasal sinuses after endoscopic skull base surgery. J Neurol Surg B Skull Base 2013;74(06): 351-357

15 Little AS, Kelly DF, Milligan J, et al. Comparison of sinonasal quality of life and health status in patients undergoing microscopic and endoscopic transsphenoidal surgery for pituitary lesions: a prospective cohort study. J Neurosurg 2015;123(03):799-807

16 Little AS, Kelly D, Milligan J, et al. Predictors of sinonasal quality of life and nasal morbidity after fully endoscopic transsphenoidal surgery. J Neurosurg 2015;122(06):1458-1465

17 Karabatsou K, O'Kelly C, Ganna A, Dehdashti AR, Gentili F. Outcomes and quality of life assessment in patients undergoing endoscopic surgery for pituitary adenomas. $\mathrm{Br} \mathrm{J}$ Neurosurg 2008;22(05):630-635

18 Tanemura E, Nagatani T, Aimi Y, Kishida Y, Takeuchi K, Wakabayashi T. Quality of life in nonfunctioning pituitary macroadenoma patients before and after surgical treatment. Acta Neurochir (Wien) 2012;154(10):1895-1902

19 Rioja E, Bernal-Sprekelsen M, Enriquez K, et al. Long-term outcomes of endoscopic endonasal approach for skull base surgery: a prospective study. Eur Arch Otorhinolaryngol 2016;273(07):1809-1817

20 Hanson M, Patel PM, Betz C, Olson S, Panizza B, Wallwork B. Sinonasal outcomes following endoscopic anterior skull base surgery with nasoseptal flap reconstruction: a prospective study. J Laryngol Otol 2015;129(Suppl 3):S41-S46

21 Harvey RJ, Malek J, Winder M, et al. Sinonasal morbidity following tumour resection with and without nasoseptal flap reconstruction. Rhinology 2015;53(02):122-128

22 Jalessi M, Jahanbakhshi A, Amini E, Kamrava SK, Farhadi M. Impact of nasoseptal flap elevation on sinonasal quality of life in endoscopic endonasal approach to pituitary adenomas. Eur Arch Otorhinolaryngol 2016;273(05):1199-1205 